INTERNATIONAL JOURNAL OF PLANT PROTECTION VOLUME 9 | ISSUE 2 | OCTOBER, 2016 | 504-509
- e ISSN-0976-6855 | Visit us : www.researchjournal.co.in

\title{
Screening of brinjal varieties against two spotted spider mite, Tetranychus. urticae (Koch) (Tetranychidae:Acari)
}

\section{MAHENDRAKUMAR B.N. AND ABHISHEK SHUKLA*}

All India Network Project on Agricultural Acarology, Department of Entomology, N.M. College of Agriculture, Navsari Agricultural University, NAVSARI (GUJARAT) INDIA

\section{ARITCLE INFO}

Received : 24.06 .2016

Revised : 26.08.2016

Accepted : 10.09.2016

\section{KEY WORDS :}

Two spotted spider mite, T. urticae, Brinjal, Screening, Varieties

*Corresponding author: Email : shuklafrs.nau@gmail.com

\section{ABSTRACT}

An experiment was carried out to screen sixteen brinjal varieties against two spotted spider mite, T. urticae under field condition. Among all the sixteen brinjal varieties Pant bahar was found moderately susceptible to spider mite attack, while the brinjal variety JBGR-1 was tolerant to spider mite attack underthe field condition. Biomorphological characters of leaf viz., hair density, leaf thickness, length and width also play important role on spider mite incidence on different brinjal varieties.

How to view point the article : Mahendrakumar B.N. and Shukla, Abhishek (2016). Screening of brinjal varieties against two spotted spider mite, Tetranychus. urticae (Koch) (Tetranychidae:Acari). Internat. J. Plant Protec., 9(2) : 504-509, DOI : 10.15740/HAS/IJPP/ 9.2/504-509. 